\title{
Papel del estrés oxidativo en el desarrollo del deterioro cognitivo y su progresión a enfermedad de Alzheimer
}

\section{Role of oxidative stress in the development of cognitive impairment and its progression to Alzheimer's disease}

\author{
CRUZ-RODRÍGUEZ, Josue †', BETANZOS CABRERA, Gabriel”, CAMACHO DÍAZ, Brenda \\ Hildeliza" y ORTIZ-RODRÍGUEZ, María Araceli*'
}

\author{
Facultad de Nutrición, Universidad Autónoma del Estado de Morelos, México. \\ "Departamento de Bioingeniería, Instituto Tecnológico y de Estudios Superiores de Monterrey, Campus-Querétaro, México. \\ "'Instituto Politécnico Nacional-Centro de Desarrollo de Productos Bióticos, Morelos, México.
}

ID $1^{\text {er }}$ Autor: Josue, Cruz-Rodríguez / ORC ID: 0000-0001-7145-5836, Researcher ID Thomson: T-3576-2018, CVU CONACYT ID: 920492

ID $1^{\text {er }}$ Coautor: Gabriel, Betanzos-Cabrera / ORC ID: 0000-0003-0847-0261, CVU CONACYT ID: 121058

ID $2^{\text {do }}$ Coautor: Brenda Hildeliza, Camacho-Díaz / ORC ID: 0000-0001-5562-0782, CVU CONACYT ID: 205272

ID $3^{\text {er }}$ Coautor: María Araceli, Ortiz-Rodríguez / ORC ID: 0000-0003-0847-0261, CVU CONACYT ID: 449164

DOI: $10.35429 /$ JOHS.2019.20.6.14.22

Recibido Julio 10, 2019; Aceptado Septiembre 19, 2019

Resumen

Esta revisión tiene como objetivo aportar evidencia científica del papel que tiene el estrés oxidativo en el desarrollo del deterioro cognitivo y su progresión a enfermedad de Alzheimer. El estrés oxidativo se origina cuando exista una producción incontrolada de radicales libres que interrumpe el equilibrio entre los oxidantes y los antioxidantes, favoreciendo a los primeros. Se ha asociado al estrés oxidativo con la patogenia del envejecimiento cerebral, el deterioro cognitivo y algunas enfermedades neurológicas. Las células del sistema nervioso central producen una alta cantidad de radicales libres ya que su demanda energética es elevada, esto aunado a una baja capacidad antioxidante, favorece la aparición de un ambiente pro-oxidante que contribuye a la neurodegeneración y muerte neuronal. La enfermedad de Alzheimer es la forma más frecuente de demencia, se caracteriza por cambios neurodegenerativos que cursan con deterioro cognitivo, afección progresiva de la memoria y el pensamiento, hasta impedir la realización de las actividades de la vida diaria. Neuropatológicamente, se caracteriza por la presencia de depósitos extracelulares de péptido $\beta$-amiloide en forma de placas y ovillos neurofibrilares; lesiones capaces de generar daño y muerte neuronal que conducen al fallo cognitivo a través de la generación de más radicales libres.

Estrés oxidativo, Deterioro cognitivo, Enfermedad de Alzheimer

\begin{abstract}
This review aims to provide scientific evidence of the role of oxidative stress in the development of cognitive impairment and its progression to Alzheimer's disease. Oxidative stress originates when there is an uncontrolled production of free radicals that disrupts the balance between oxidants and antioxidants, favoring oxidants. It has been associated with oxidative stress with the pathogenesis of brain aging, cognitive impairment and some neurological diseases. The cells of the central nervous system produce a high amount of free radicals since their energy demand is high, this coupled with a low antioxidant capacity, favors the appearance of a pro-oxidant environment that contributes to neurodegeneration and neuronal death. Alzheimer's disease is the most frequent form of dementia, it is characterized by neurodegenerative changes that occur with cognitive impairment, progressive impairment of memory and thought, until preventing the performance of daily life activities. Neuropathologically, it is characterized by the presence of extracellular deposits of $\beta$-amyloid peptide in the form of neurofibrillar plaques and clews; lesions capable of generating damage and neuronal death that lead to cognitive failure through the generation of more free radicals.
\end{abstract}

Oxidative stress, Cognitive impairment, Alzheimer's disease

Citación: CRUZ-RODRÍGUEZ, Josue, BETANZOS CABRERA, Gabriel, CAMACHO DÍAZ, Brenda Hildeliza y ORTIZRODRÍGUEZ, María Araceli. Papel del estrés oxidativo en el desarrollo del deterioro cognitivo y su progresión a enfermedad de Alzheimer. Revista de Ciencias de la Salud. 2019. 6-20: 14-22.

*Correspondencia al Autor (Correo Electrónico: araceli.ortiz@gmail.com)

$\uparrow$ Investigador contribuyendo como primer Autor 


\section{Introducción}

El estrés oxidativo se origina cuando exista una producción incontrolada de radicales libres que interrumpe el equilibrio oxidante-antioxidante a favor de los compuestos oxidantes, propiciando la aparición de alteraciones fisiológicas que conducen a la enfermedad. Se ha implicado al estrés oxidativo en la patogenia del envejecimiento cerebral, el deterioro cognitivo y algunas enfermedades neurológicas (Viada et al., 2017; Rodríguez et al., 2015; Sies et al., 2017; Pisoschi et al., 2015).

En los últimos años se han presento diversos estudios que sugieren una participación importante del estrés oxidativo en la aparición del deterioro cognitivo y su progresión a enfermedad de Alzheimer. Entre ellos se encuentran el aumento en la oxidación de lípidos, proteínas y ácidos nucleicos, la acumulación del péptido $\beta$-amiloide que favorece la formación de placas seniles e induce una respuesta inflamatoria local, asociadas directamente al proceso lesivo neuronal por ser grandes fuentes de radicales libres de oxígeno, contribuyendo a aumentar el estrés oxidativo y sus efectos neurodegenerativos (Viada et al., 2017; Zafrilla et al., 2004; Gandhi y Abramov, 2012).

\section{Estrés oxidativo}

La oxidación es todo proceso en el que ocurre pérdida de electrones de hidrógeno y ganancia de oxígeno, mientras que la reducción es la ganancia de electrones de hidrógeno y pérdida de oxígenos. De esta manera la oxidación de una molécula siempre estará acompañada de un proceso de reducción de otra molécula, en conjunto a estos dos procesos se les conoce como reacciones redóx o reacciones de óxidoreducción (Rodríguez et al., 2015; Coronado et al., 2015; Botham et al., 2016).

Estas reacciones de óxido reducción son imprescindibles para todos los seres vivos ya que forman parte de los procesos fundamentales de la vida, uno de los cuales es el metabolismo aeróbico, mediante el cual se produce la mayor cantidad de energía libre.
El metabolismo aeróbico requiere indispensablemente del oxígeno (O2) para desarrollar sus funcionas, entre más oxígeno haya disponibilidad mayor será la oxidación de los compuestos orgánicos y mayor liberación de energía en forma de Adenosín trifosfato (ATP), (Rodríguez et al., 2015; Sies et al., 2017). El oxígeno es un elemento químico de naturaleza gaseosa y es fundamental para el mantenimiento de la vida, sin embargo puede llegar a causar enfermedad debido a su capacidad de formar compuestos y a su naturaleza radical, ya que en su estructura química presenta dos electrones no pareados en el último orbital, ambos electrones presentan un giro paralelo que impide la captación simultanea de dos electrones ya que la donación simultanea de dos electrones siempre es anti paralela, debido a esto la molécula de oxígeno solo puede aceptar un electrón, lo que la transforma en un radical (Viada et al., 2017; Rodríguez et al., 2015; Sies et al., 2017; Pisoschi et al., 2015).

\section{Radicales libres}

Los radicales libres son átomos o moléculas, con uno a más electrones desapareados en su orbital más externo. Son altamente inestables, reactivos y de vida muy corta (microsegundos), son capaces de reaccionar con otras biomoléculas atreves de su oxidación, formando más radicales libres mediante reacciones en cadena llegando a causar gran daño puesto que un radical libre es capaz de afectar hasta un millón de moléculas cercanas (Coronado et al., 2015; Sánchez y Méndez, 2013).

La formación de radicales libres se da a través de distintos mecanismos, la causa principal son las alteraciones en la respiración aeróbica mitocondrial, el metabolismo de los alimentos, mala calidad de la alimentación, consumo de tabaco, ingesta de alcohol, drogas, fármacos y estupefacientes, ejercicio intenso, estrés físico o psicológico, enfermedades preexistentes, exposición a contaminantes ambientales (ozono, hipoclorito, monóxido de carbono, óxido nitroso, dióxido de azufre, etc.), xenobióticos (fertilizantes, herbicidas, pesticidas, etc.), radiaciones (luz ultravioleta, rayos $\mathrm{X}$ y rayos gamma), aumento en los niveles de algunos iones metálicos (hierro, cobre, cadmio, cromo, niquel, mercurio y otros), etc. (Viada et al., 2017; Maldonado et al., 2010). 


\section{Clasificación de los radicales libres}

Los radicales libres se clasifican de acuerdo al grupo funcional que los conforma, el grupo de mayor importancia son las especies reactivas de oxígeno (ROS), este grupo incluye al radical superóxido, hidroxilo y alcoxilo, peróxido de hidrógeno, hidroperoxilo, ácido hipocloroso y oxígeno singlete, entre otros. Otros grupos son las especies reactivas de nitrógeno (RNS) que incluye al óxido nítrico, acido nitroso, dióxido de nitrógeno, peroxinitrito y el ácido peroxinitroso, las especies reactivas de azufre (RSS) que incluye todas las isoformas de metionina y cisteína, las especies de carbonilos reactivos (RCS) conformada por carbonilos y aldehídos generados en el metabolismo y las especies reactivas de selenio (RSE) que agrupan a la selenocisteína y selenometionina (Viada $e t$ al., 2017; Sies et al., 2017; Coronado et al., 2015).

\section{Especies reactivas de oxígeno}

Debido al papel fundamental que tiene el oxígeno en los procesos aerobios y en la preservación de la vida, es considerado el mayor productor de ROS, este grupo incluye tanto derivados radicales como no radicales de oxígeno (Viada et al., 2017; Sies et al., 2017; Corrales y Muñoz, 2012).

Radical superóxido: Es el radical más abundante en la célula, se forma por reducción del oxígeno, principalmente en la cadena de transporte de electrones y el citocromo P450. También se genera mediante la oxidación de la hemoglobina, ubiquinona, catecolaminas, enzima NADPH oxidasa y xantina oxidasa. Es relativamente inestable y por medio de él se pueden producir casi todas los demás ROS (Pisoschi et al., 2015).

Radical hidroxilo: Es una de los ROS más reactivas y dañinas del organismo, resulta de la reducción del oxígeno molecular o de la reacción entre el anión superóxido y el peróxido de hidrógeno catalizada por iones metales, su vida media es muy corta, su electrón no pareado es capaz de reaccionar con cualquier tipo de molécula, las células no cuentan con un sistema antioxidante para contrarrestar los efectos de este radical (Gutiérrez-Salinas et al., 2014; Pedraza y Cárdenas, 2019).
Radical peroxilo y alcoxilo: Resultan de las reacciones entre radicales y átomos de carbono u oxígeno, su fuente principal es la ruptura de peróxidos orgánicos o lipoperoxidación, son menos tóxicos y reactivos que los anteriores, debido a ello su vida media es un poco mayor, sin embargó su reacción en cadena aumenta la peroxidación de biomoléculas (Camps et al., 2010).

Hidroperoxilo: Es la forma protonada y dismutada del anión superóxido, su vida media y potencial oxidante es mayor al del anión superóxido, es capaz de iniciar y terminar el proceso de lipoperoxidación (Camps et al., 2010).

Oxígeno singlete: Es la forma excitada energéticamente del oxígeno, con dos electrones pareados en los orbitales de energía mayor. Se produce en reacciones catalizadas por oxidasas y durante la fagocitosis, es capaz de oxidar algunos aminoácidos como el triptófano y la metionina causando daño a las proteínas y al ADN, favorece el inicio de la lipoperoxidación originando nuevas ROS. Su vida media es muy corta y no tiene restricciones al momento de realizar transferencia de electrones (Sies et al., 2017; Gutiérrez-Salinas et al., 2014; Pedraza y Cárdenas, 2019).

Peróxido de hidrógeno: Se forma mediante la dismutación del anión superóxido por la enzima superóxido dismutasa, su potencial oxidante y reductor es débil, sin embargo, en presencia de metales de transición puede reaccionar con el anión superóxido y producir radicales altamente reactivos como el radical hidroxilo (Pisoschi et al., 2015; Pedraza y Cárdenas, 2019; Camps et al., 2010).

Ácido hipocloroso: Se produce de la unión del óxido de cloro y agua o por la acción de la enzima mieloperoxidasa que cataliza la conversión del peróxido de hidrógeno en ácido hipocloroso por oxidación de iones cloro. En bajas concentraciones daña las proteínas de membrana mediante la oxidación de los grupos sulfhidrilo, mientras que en altas concentraciones causa daño generalizado a la célula provocando que la lisis celular (Pisoschi et al., 2015; Pedraza y Cárdenas, 2019; Camps et al., 2010). 


\section{Efectos químicos y biológicos de las ROS}

Las ROS son muy reactivas y se producen constantemente en el organismo, al existir una gran cantidad de ellos se desarrolla un ambiente pro-oxidante, generando daño a distintas moléculas del organismo y liberando productos finales que poseen mayores efectos citotóxicos (Corrales y Muñoz, 2012; Pedraza y Cárdenas, 2019).

\section{Efectos sobre los hidratos de carbono}

Algunos glúcidos como los monosacáridos y disacáridos son resistentes a la acción de ROS, mientras que los polisacáridos pueden ser despolimerizados. Aunque la glucosa puede capturar al anión superóxido e impedir que actué sobre otras moléculas, cuando existe hiperglucemia crónica, la glucosa puede reaccionar con los grupos amino de las proteínas formando productos altamente reactivos, resultado de la glicación no enzimática de las proteínas (compuestos amadori), estos productos tienen la capacidad de reducir al oxígeno y formar ROS. Además, la prolongación de la glicación no enzimática de proteínas genera productos de glucoxidación avanzada, los cuales se acumulan en los tejidos con la edad y causan aumento en el nivel de glucosa y estrés oxidativo (Sies et al., 2017; Maldonado et al., 2010).

\section{Efectos de las ROS sobre los lípidos}

El principal efecto de las ROS sobre los lípidos es la lipoperoxidación o peroxidación de los ácidos grasos poliinsaturados, esta reacción se inicia por la captura de un átomo de hidrógeno de uno de los carbonos de un ácido graso, dejando un electrón desapareado, de esta manera se genera un radical lipídico que al reaccionar con el oxígeno molecular forma un radical hidroperoxil, radical que a su vez captura un átomo de hidrógeno de un carbono de otro ácido graso poliinsaturado, formando otro radical lipídico y un hidroperóxido lipídico, este radical lipídico reacciona con otra molécula de oxígeno, originando una reacción en cadena (Muñiz et al., 2014; Sies et al., 2017; Pisoschi et al., 2015; Pedraza y Cárdenas, 2019). Entre los productos finales de la lipoperoxidación se encuentra el maondialdehíco, que puede causar daño a las proteínas y al ADN debido a su alto poder citotóxico.
La peroxidación trae como consecuencia alteraciones en la membrana, pérdida de la flexibilidad que afecta fluidez y provocando daño en su estructura. Se ha relacionado a la lipoperoxidación con el desarrollo de enfermedades cardiovasculares ya que oxida a las proteínas de baja densidad (Sies et al., 2017; Pisoschi et al., 2015; Pedraza y Cárdenas, 2019).

\section{Efectos de las ROS sobre las proteínas}

La acción de las ROS sobre las proteínas depende de los aminoácidos que la conforman, actúan sobre los anillos aromáticos y los puentes disulfuro, de modo que las proteínas ricas en fenilalanina, histidina, triptófano, metionina y tirosina son más susceptibles a la actividad de las ROS. El anión superóxido e hidroperóxido rompen las fibrillas de colágeno favoreciendo la acción de las proteasas y la perdida de la estructura de triple hélice de las proteínas induciendo una esclerosis y fibrosis del tejido de sostén que pierde su tonicidad (aparición de arrugas). El peróxido de hidrógeno en presencia de algunas peroxidasas es capaz de oxidar al ion cloruro para formar ácido hipocloroso que es altamente toxico para bacterias y virus, sin embargo, en altas concentraciones daña y lisa la célula. Los efectos de las ROS sobre las proteínas se han asociado con enfermedades como la artritis reumatoide y el Alzheimer (Sies et al., 2017; Pisoschi et al., 2015; Pedraza y Cárdenas, 2019).

\section{Efectos sobre los ácidos nucleicos}

Los ácidos nucleicos son particularmente vulnerables a la acción de las ROS, actúan entre las bases púricas y pirimídicas por hidroxilación de bases nitrogenadas, escisión de las hebras de ADN (ruptura del esqueleto azúcar fosfato) y formación de uniones cruzadas (ADN-ADN), causando alteraciones en los procesos de replicación y transcripción del ADN que resultan en mutaciones que pueden causar cáncer, apoptosis o necrosis (Sies et al., 2017; Pisoschi et al., 2015; Pedraza y Cárdenas, 2019).

\section{Antioxidantes}

Puesto que los radicales libres se producen de forma constante en el organismo, las células han desarrollado poderosos sistemas de defensa antioxidante. 
El concepto de antioxidante se utiliza, para referirse a cualquier compuesto químico que, cuando se encuentra a concentración más baja que la de un sustrato oxidable, puede retrasar o evitar su oxidación, previniendo la formación descontrolada de radicales libres y el daño sobre biomoléculas (Viada et al., 2017; Coronado et al., 2015; Pisoschi et al., 2015).

\section{Mecanismos de acción antioxidantes}

Los antioxidantes actúan en diferentes etapas de la acción de los radicales libres. Actúan disminuyendo o agotando la concentración local de oxígeno, capturando al anión superóxido o a él peróxido de hidrógeno, eliminando a los radicales hidroxilo, alcoxilo o peroxil, apagando al oxígeno singlete y eliminando aniones metálicos que promueven la oxidación. De acuerdo a ello se dividen en dos grupos: (Pisoschi et al., 2015; Pedraza y Cárdenas, 2019).

Preventivos: Son aquellos que actúan al inicio de la cadena oxidativa, inhiben la peroxidación lipídica, disminuyen las concentraciones de oxígeno, eliminan los iones metales de transición o agotan catalíticamente a los radicales libres (Pisoschi et al., 2015; Pedraza y Cárdenas, 2019).

Secundarios: Actúan bloqueando la reacción en alguna etapa de la cadena oxidativa, mediante agotamiento del oxígeno singlete, descomponiendo peróxidos o por acción de quelantes metálicos (Pisoschi et al., 2015; Pedraza y Cárdenas, 2019).

Los antioxidantes secundarios actúan en combinación con los antioxidantes primarios, mediante 4 mecanismos (Pisoschi et al., 2015; Pedraza y Cárdenas, 2019):

1. Estabiliza los antioxidantes primarios creando un ambiente ácido.

2. Regenerando antioxidantes primarios por donación de hidrógeno.

3. Cationes de metales de transición quelantes.

4. Enfriamiento del oxígeno molecular.
Las interacciones entre antioxidantes, metabolitos y sistemas enzimáticos, potencia los mecanismos de acción antioxidante, sin embargo, la protección brindada por cualquier antioxidante depende de su concentración y su reactividad hacia las ROS (Viada et al., 2017).

\section{Clasificación de los antioxidantes}

Los antioxidantes se pueden dividirse en dos grandes grupos de moléculas:

Antioxidantes enzimáticos: aquellas que tienen una estructura compleja y elevado peso molecular. Cataliza la transferencia de electrones desde un sustrato hacia los radicales libres (Pisoschi et al., 2015; Sánchez y Méndez, 2013; Corrales y Muñoz, 2012; Pedraza y Cárdenas, 2019; Mayor, 2014).

Antioxidantes no enzimáticos: Son un grupo heterogéneo de moléculas hidrófobas e hidrófilas, de menor tamaño y peso molecular, tienen como función; capturar al radical libre y producir moléculas menos nocivas, mediante la adición de un electrón al radical libre (Pisoschi et al., 2015; Sánchez y Méndez, 2013; Corrales y Muñoz, 2012; Pedraza y Cárdenas, 2019; Mayor, 2014).

\section{Antioxidantes enzimáticos}

Superóxido Dismutasa (SOD): Es una enzima localizada en el citosol y en la mitocondria, su función es catalizar la conversión del anión superóxido a peróxido de hidrógeno. Se pueden encontrar 2 isoformas (Gandhi y Abramov, 2012; Pisoschi et al., 2015; Pedraza y Cárdenas, 2019):

- Sod1 (SodCuZn): Es un dímero de cobre y zinc unido a la superóxido dismutasa y se encuentra presente en el citosol.

- Sod2 (Sodmn): Es un tetrámero que contiene manganeso en su sitio activo y se localiza en la mitocondrial.

Glutatión peroxidasa (GPx): Es una enzima citosólica que contiene selenio, se encarga de eliminar el peróxido de hidrógeno y algunos hidroperóxidos, actuando en conjunto con el glutatión reducido y se puede encontrar en 4 isoformas (Gandhi y Abramov, 2012; Pisoschi et al., 2015; Pedraza y Cárdenas, 2019): 
- GPx celular: Se encuentra en todas las células y se encarga de reducir el peróxido de hidrógeno e hidroperóxidos en agua y alcohol.

- GPx extracelular: Se sintetiza en las células proximales del riñón.

- GPx fosfolípido hidroperóxido: Previene la lipoperoxidación, reduciendo los ácidos grasos de las membranas celulares.

- GPx gastrointestinal: Da protección contra la toxicidad generada por el consumo de hidroperóxidos lipídicos.

Glutatión reductasa (GRs): Enzima citoplasmática que en su sitio activo contiene coenzima FAD. Se encarga de catalizar la reducción del glutatión disulfuro en dos moléculas de glutatión (Pedraza y Cárdenas, 2019).

Catalasa: Esta enzima se localiza en el citosol y en los organelos celulares (peroxisomas y mitocondrias), que elimina peróxido de hidrógeno descomponiéndolo en agua y oxígeno. Está presente en la mayoría de las células, órganos y tejidos (Gandhi y Abramov, 2012; Pisoschi et al., 2015; Pedraza y Cárdenas, 2019).

Tiorredoxina (Trx): Pertenece al grupo de los tioles que son amotiguadores antioxidantes, es un polipéptido que mantiene la homeostasis intracelular, es capaz de donar electrones en diversas reacciones de oxidación y reducción (Pisoschi et al., 2015; GutiérrezSalinas et al., 2014).

\section{Antioxidantes no enzimáticos}

Vitamina C: También conocida como ácido ascórbico, se encuentra tanto intra como extracelularmente, actúa directamente sobre el anión superóxido, hidroxilo, alcoxilo e hidroperóxidos lipídicos. Participa en el mantenimiento de la integridad del tejido conjuntivo y vascular, en la absorción de hierro, la hematopoyesis y la neuroprotección (elimina radicales libres en el cerebro), también protege a los fosfolípidos de membrana del daño peroxidativo (Pisoschi et al., 2015; GutiérrezSalinas et al., 2014).
La actividad del ácido ascórbico como antioxidante primario en plasma es la mayor, sin embargo, actúa como pro-oxidante en presencia de metales de transición (Pisoschi et al., 2015; Gutiérrez-Salinas et al., 2014).

Vitamina E: También conocido como alfa-tocoferol, tiene la capacidad de contrarrestar la lipoperoxidación en la membrana celular. Se ha visto que la ingesta a dosis altas de vitamina $\mathrm{E}$ inhibe los procesos proaterogénicos y mejora los niveles de biomarcadores de estrés oxidativo (Gandhi y Abramov, 2012; Pisoschi et al., 2015).

Carotenoides: Los carotenoides reaccionan con los radicales libres mediante transferencia de electrones, extracción de hidrógeno y adición de radicales. El $\beta$ caroteno y el licopeno son inhibidores del oxígeno singlete, de igual forma el licopeno es un potente eliminador de radicales peroxilo (Pisoschi et al., 2015).

Flavonoides polifenólicos: Este grupo comprende a los ácidos fenólicos y los flavonoides que son compuestos que actúan como quelantes de metales y son capaces de atrapar ROS. Se ubican intra y extracelularmente, pueden ser liposolubles como hidrosolubles (Pisoschi et al., 2015; GutiérrezSalinas et al., 2014).

Ácido Úrico: Es un potente eliminador de radicales singlete de oxígeno, peroxilo e hidroxilo, se encarga de proteger a la membrana eritrocitaria de la lipoperoxidación. En presencia de iones de cobre e hidroperóxidos se convierte en un pro-oxidante (Pisoschi et al., 2015).

\section{Estrés oxidativo y deterioro cognitivo}

La fisiopatología de las enfermedades neurodegenerativas no ha sido descrita del todo a pesar de las investigaciones realizadas en este campo, sin embargo, algunos de estos estudios colocan al estrés oxidativo como un factor principal en el desarrollo de estas patologías. 
Las células del sistema nervioso central producen una alta cantidad de radicales libres ya que su demanda energética es elevada, esto aunado a una baja capacidad antioxidante, favorece la aparición de un ambiente prooxidante y el desarrollo de estrés oxidativo que contribuye a la muerte celular, neurodegeneración y pérdida de neuronas (Rodríguez et al., 2015; Gandhi y Abramov, 2012).

La enfermedad de Alzheimer es la forma más frecuente de demencia, se caracteriza por cambios neurodegenerativos que cursan con deterioro cognitivo y afección progresiva de la memoria y el pensamiento, hasta impedir la realización de las actividades de la vida diaria (Borrell, 2017; Persson et al., 2014). Neuropatológicamente, se caracteriza por la presencia de depósitos extracelulares de péptido $\beta$-amiloide en forma de placas y ovillos neurofibrilares. Estas lesiones son capaces de generar daño y muerte neuronal que conducen al fallo cognitivo a través de la generación de ROS (Luque-Contreras et al., 2014).

Las mitocondrias son los orgánulos responsables de proveer a la célula la energía necesaria para realizar sus procesos metabólicos en condiciones aerobias. En las neuronas la función de las mitocondrias es de gran importancia, ya que sus necesidades energéticas son mayores en comparación con otras células del cuerpo y de este aporte de energía depende el funcionamiento de la maquinaria sináptica (Persson et al., 2014; Luque-Contreras et al., 2014).

La disfunción mitocondrial se ha observado como característica de muchas enfermedades neurodegenerativas, entre ellas el deterioro cognitivo y la enfermedad de Alzheimer. Los defectos en la función mitocondrial dañan la célula de dos maneras, uno es mediante el aumento significativo de la producción y liberación de ROS, que a su vez causan daño y muerte celular y el otro mediante agotamiento de la energía por interrupción de la oxidación para producir ATP. Estudios recientes muestran que el péptido $\beta$-amiloide puede ser responsable de la muerte neuronal y la pérdida de sinapsis debido a los efectos adversos que tiene sobre la estructura y función mitocondrial (Persson et al., 2014; Luque-Contreras et al., 2014).
El agotamiento de los fosfolípidos de las membranas, como consecuencia de la lipoperoxidación, se ha descrito como causa principal de la aparición del deterioro cognitivo y la enfermedad de Alzheimer. Las neuronas son particularmente vulnerables a los efectos de los radicales libres y la lipoperoxidación por su alta concentración de ácidos grasos poliinsaturados (Pisoschi et al., 2015). El péptido $\beta$-amiloide es capaz de comenzar la lipoperoxidación, aumentando la producción de peróxidos lipídicos y la disminución de la actividad antioxidante, que favorece la formación de nuevas placas seniles y neurofibrilares en los sujetos con deterioro cognitivo y enfermedad de Alzheimer. Otro proceso de importancia en la progresión del deterioro cognitivo es la carbonilación de las proteínas de membrana en el hipocampo y la corteza parietal y frontal del cerebro, ya que causa daño en el transportador neuronal de glucosa GLUT-3, los transportadores de glutamato, las ATPasas sodio/potasio y la homeostasis del calcio, propiciando una mayor producción de ROS y muerte celular, que aumentan los procesos de neurodegeneración (Pisoschi et al., 2015).

La formación de placas seniles por acumulación de péptido $\beta$-amiloide en el cerebro, es el factor más importante en la aparición del deterioro cognitivo y su progresión en la enfermedad de a Alzheimer. Se ha visto que la acumulación de péptido $\beta$-amiloide provoca un efecto neurotóxico caracterizado por la presencia de estrés oxidativo, daño a las membranas, al ADN mitocondrial y a los lípidos (Persson et al., 2014; Luque-Contreras et al., 2014).

Los péptidos $\beta$-amiloides poseen entre 39 y 42 residuos de aminoácidos, siendo el péptido $\beta$-amiloide 40 el más producido y 42 el menos producido. No obstante, la relación entre ambos péptidos en la enfermedad de Alzheimer es de 50/50\% (Persson et al., 2014; Luque-Contreras et al., 2014). Los oligómeros de amiloide son la forma más toxica de la proteína y son capaces de insertarse en la bicapa lipídica causando lipoperoxidación y en consecuencia daño oxidativo a las proteínas y otras biomoléculas. Como resultado de la alteración en la membrana, hay una afluencia masiva de calcio, que altera sus concentraciones y causa disfunción mitocondrial, pérdida de sinapsis y muerte neuronal (Persson et al., 2014; Luque-Contreras et al., 2014). 
Otra proteína importante en la progresión del deterioro cognitivo a enfermedad de Alzheimer es la apolipoproteína E (ApoE) que participa en el mantenimiento de la integridad estructural de las membranas y correcto funcionamiento de la sinapsis. Existen 3 isoformas de ApoE: ApoE2, ApoE3 y ApoE4. Siendo los sujetos que portan la isoforma ApoE4 los que presentan un mayor riesgo para desarrollar la enfermedad de Alzheimer, debido a que la ApoE4 presenta mayor afinidad con la proteína precursora de $\beta$-amiloide, lo que aumenta su producción y la formación de placas seniles (Persson et al., 2014; Luque-Contreras et al., 2014).

Mientras que la isoforma ApoE2 posee efectos antioxidantes y ofrece mayor protección contra la $\beta$-toxicidad que las isoformas ApoE3 y ApoE4, lo que sugiere que la presencia de ApoE4 favorece la pérdida del equilibrio antioxidante y la progresión del deterioro cognitivo a enfermedad de Alzheimer (Persson et al., 2014; Luque-Contreras et al., 2014).

\section{Conclusión}

La prevalencia de enfermedades neurodegenerativas es un problema de salud pública de gran importancia, en México el 7.9\% de los adultos mayores de 60 años padece demencia. En los últimos años se han realizado diferentes investigaciones sobre la fisiopatología del deterioro cognitivo y la enfermedad de Alzheimer, presentado resultados que sugieren un papel importante del estrés oxidativo en la patogenia de estas enfermedades. Entre ellos se incluyen alteraciones en las concentraciones de factores oxidantes y antioxidantes en el cerebro, la lipoperoxidación, la oxidación de las proteínas, alteraciones de la función mitocondrial y la acumulación de péptido $\beta$ amiloide, que en conjunto favorecen la producción de nuevos radicales libres causando, causando daño neurológico. Sin embargo, aún no se han logrado establecer los mecanismos exactos de la fisiopatología de estas enfermedades, por lo que se les deben seguir realizando estudios que permitan identificarlos.

\section{Agradecimientos}

Josué Cruz Rodríguez fue financiado por una beca de PNPC de CONACYT.

\section{Referencias}

Borrell, F. (2017). Enfermedad de Alzheimer y factores de riesgo ambientales. Revista Cubana de Enfermería, 33(1).

Botham, K., \& Mayes, P. Oxidación biológica. En: Rodwell, V., Bender, D., Botham, K., \& Kennelly, P. (2016). Bioquímica de Harper. 30 a edición. España. Mc Graw Hill interamericana, 119-125.

Camps, D., Ruffino, S., Majul, E., \& Joison A. (2010). Bioquímica del estrés oxidativo y de las especies reactivas de oxígeno. Primera edición. Editorial Lulu.

Coronado, M., Vega y León, S., Gutiérrez, R., Vázquez, M., \& Radilla, C. (2015). Antioxidantes: perspectiva actual para la salud humana. Revista chilena de nutrición, 42(2), 206-212.

Corrales, L., \& Muñoz, M. (2012). Estrés oxidativo: origen, evolución y consecuencias de la toxicidad del oxígeno, Nova, 10(18), 135-225.

Gandhi, S., \& Abramov, A. Y. (2012). Mechanism of oxidative stress in neurodegeneration. Oxidative medicine and cellular longevity, 2012, 428010.

Gutiérrez-Salinas, J., Mondragón-Terán, P., García-Ortíz, L., Hernández-Rodríguez, S., Ramírez-García, S., \& Núñez-Ramos, N. R. (2014). Breve descripción de los mecanismos moleculares de daño celular provocado por los radicales libres derivados de oxígeno y nitrógeno. Revista de Especialidades MédicoQuirúrgicas, 19(4), 446-454.

Luque-Contreras, D., Carvajal, K., Toral-Rios, D., Franco-Bocanegra, D., \& Campos-Peña, V. (2014). Oxidative stress and metabolic syndrome: cause or consequence of Alzheimer's disease?. Oxidative medicine and cellular longevity, 2014, 497802.

Maldonado, O., Jiménez, E., Guapillo, M., Ceballos, G., \& Méndez, E. (2010). Radicales libres y su papel en las enfermedades crónicodegenerativas. Revista Médica de la Universidad Veracruzana, 10(2), 32-39. 
Mayor, R. (2014). Estrés oxidativo y sistema de defensa antioxidante. Revista Del Instituto de Medicina Tropical, 5(2), 23-27.

Muñiz, P., Coma, M.J., \& Terán, J. (2014). Estrés oxidativo y daño vascular en procesos de hipoxia. Malondialdehido (MDA) como biomarcador de daño oxidativo. Electronic Journal of Biomedicine, 2, 46-49.

Pedraza, J., \& Cárdenas N. (2006). Especies reactivas de oxígeno y sistemas antioxidantes. Aspectos básicos. Educacion química, 17(2), 164-173.

Persson, T., Popescu, B. O., \& Cedazo-Minguez, A. (2014). Oxidative stress in Alzheimer's disease: why did antioxidant therapy fail?. Oxidative medicine and cellular longevity, 2014, 427318.

Pisoschi, A. M., \& Pop, A. (2015). The role of antioxidants in the chemistry of oxidative stress: A review. European journal of medicinal chemistry, 5(97), 55-74.

Rodríguez, T., Peña, M., Gómez, N., Santisteban, Y., \& Hernández, M. (2015). Estrés oxidativo: genética, dieta y desarrollo de enfermedades. Correo Científico Médico, 19(4), 690-705.

Sánchez-Valle, V., \& Méndez-Sánchez, N. (2018). Estrés oxidativo, antioxidantes y enfermedad. Médica Sur, 20(3), 161-168.

Sies, H., Berndt, C., \& Jones, D. P. (2017). Oxidative stress. Annual review of biochemistry, 20(86), 715-748.

Viada, E., Robles, L., \& Marrero, I. (2017). Oxidative stress. Correo Científico Médico, 21(1), 171-186.

Zafrilla, P., Morillas, J., Mulero, J., Xandri, J. M., Santo, E., \& Caravaca, G. (2004). Estrés oxidativo en enfermos de Alzheimer en diferentes estadios de la enfermedad. Nutrición Hospitalaria, 19, 63-63. 